\title{
3D Characterization of Nanoparticle Dispersion in Polymer Nanocomposites by TEM Tomography.
}

Florent Dalmas ${ }^{1}$, Eric Leroy ${ }^{1}$, Matthias Roth ${ }^{1}$, Fatma Allouche ${ }^{1}$, François Boué ${ }^{2}$, Fabrice Cousin $^{2}$ and Jacques Jestin ${ }^{2}$

1. ICMPE (Institut de Chimie et des Matériaux Paris-Est), UMR 7182 CNRS/Université Paris-Est Créteil, 2-8 rue Henri Dunant, 94320 Thiais, France

2. LLB (Laboratoire Léon Brillouin), CEA/CNRS, CEA Saclay 91191 Gif-sur-Yvette Cedex, France

In polymer-based nanocomposites, the macroscopic properties are driven by one specific feature: the huge interfacial area developed by nanofillers, leading to a strong improvement in mechanical, thermal or electrical properties. Even though the molecular origins of this reinforcement are still not well understood, its amplitude appears to be strongly related to two main effects: a structural effect (shape, dispersion and organisation of the nanofillers within the material) and an interfacial effect (nature and strength of the filler-filler and filler-matrix interactions). Despite the numerous studies that investigated the influence of specific parameters on the behavior of nanocomposites, it is still difficult to determine the respective role of these two effects [1]. Consequently, the understanding of the relationships between the microstructure and the mechanical properties is still an open question. On the other hand, Transmission Electron Microscopy Tomography (TEMT) appeared in the last decade to be an effective and meaningful tool for three-dimensional (3D) microstructural characterization when applied to nanostructured polymeric systems [2]. In the present study we thus propose a quantitative 3D characterization of model nanocomposites in order to go further in the comprehension of this fundamental issue.

In recent studies, we investigated the synthesis, characterization by small angle scattering (neutron and $\mathrm{X}$-ray) and mechanical properties of polymeric nanocomposites consisting in a polystyrene matrix filled with various volume fractions of spherical nanoparticles (made of ungrafted or grafted silica, $\mathrm{SiO}_{2}$, or magnetic maghemite, $\gamma-\mathrm{Fe}_{2} \mathrm{O}_{3}$ ). A wide range of microstructures was obtained (see Figure 1): isolated or aggregated nanoparticles (fractal or dense aggregates were obtained), percolating structures [3-4] and anisotropic structures prepared from magnetic nanoparticles under a magnetic field [5]. In a first step, we developed in the present study a quantitative microstructural analysis based on TEMT reconstructions in order to extract relevant characteristic parameters from these microstructures. Several points are discussed regarding the type (nature, size and surface properties) and volume fraction of nanoparticles:

- Heterogeneity in the nanoparticle dispersion: anisotropy, aggregation...

- Percolation: determination of the percolation threshold, percolating backbone, isolated aggregates and connectivity of the structure (3D, 2D or 1D).

- Isolated aggregate morphology: size, inter-particle and inter-aggregate distances, geometry (specific surface, fractal dimension, density...).

In a second step, by coupling TEMT image analysis and scattering results, which is barely done in the literature, this approach allows precisely addressing different important effects in the science of polymeric nanocomposite materials. First of all, a challenging issue is the effective link between the microstructural percolation and the divergence observed in the mechanical properties in the case of ungrafted silica nanoparticles (see Figure 1(C)) [3]. Then, when grafted with polystyrene chains, these 
particles can either be perfectly dispersed or densely agglomerated within the matrix, depending on the molar mass of the grafting chains (see Figure 1(A-B)). The attractive/repulsive interparticle interactions could be discussed by analyzing the closest approach distance between nanoparticles. In the case of magnetic nanoparticles (see Figure 1(D)), the anisotropy of the structure is strongly related to the magnetic field and the size of the nanoparticles [5]. The local orientation of the aggregates could be related to the anisotropy observed in the scattering data and in the mechanical properties. To finish, some of the previous systems, strained at different elongation rate, were observed and analyzed. The correlation between the local deformation (changing in the aggregate size and anisotropy, the interparticle and inter-aggregate distances, fractal dimension of the structure...) and the macroscopic one will be discussed.

\section{References:}

[1] J Jancar et al, Polymer 51 (2010), 51, p. 3321.

[2] H Jinnai, RJ Spontak and T. Nishi, Macromolecules 43 (2010), p. 1675.

[3] N Jouault et al, Macromolecules 42 (2009), p. 2031.

[4] C Chevigny et al, Macromolecules 44 (2011), p. 122.

[5] AS Robbes et al, Macromolecules 44 (2011), p. 8858.

(A)

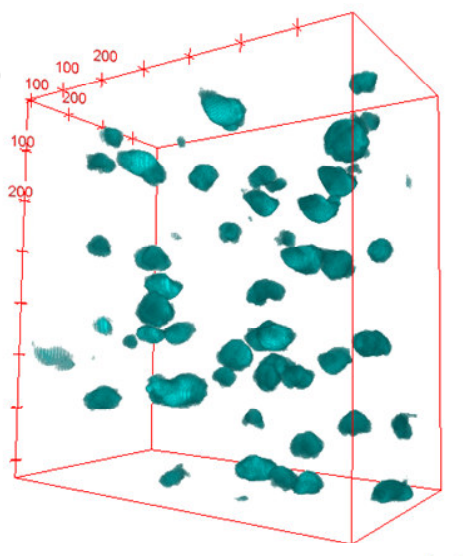

(C)

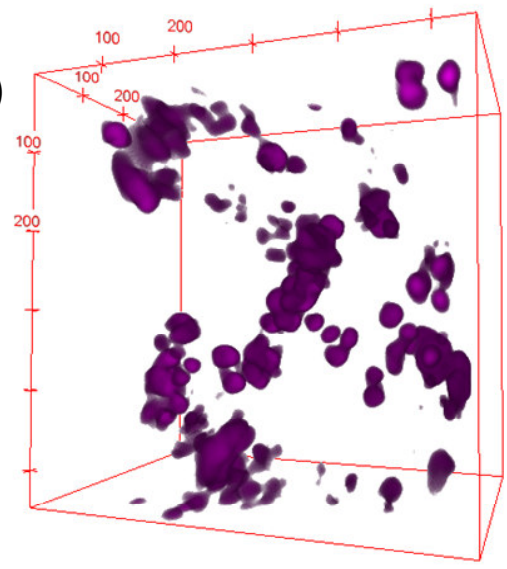

(B)
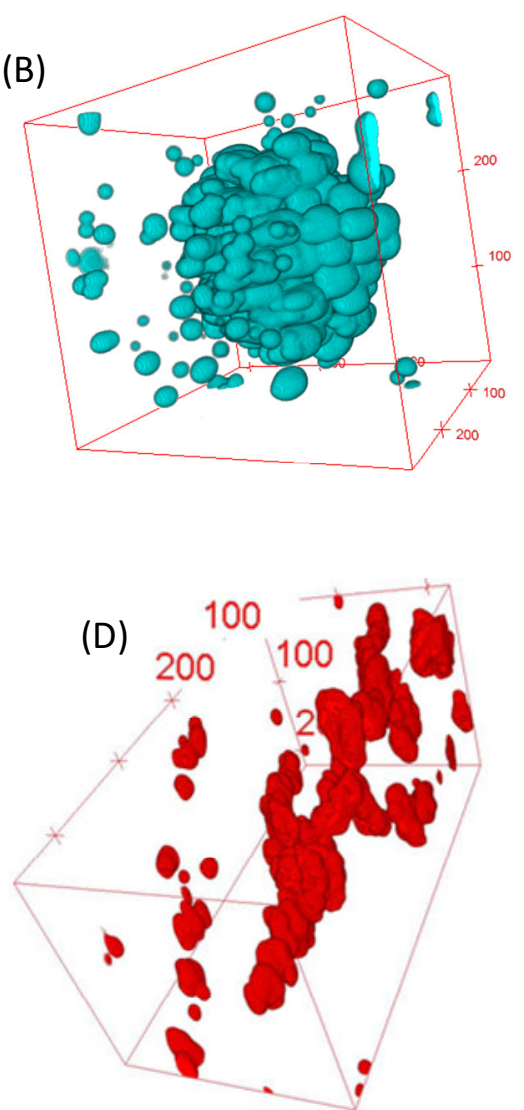

Figure 1. High magnification TEMT volume reconstructions of nanocomposites made of a polystyrene matrix reinforced with grafted silica nanoparticles either isolated (A) or densely agglomerated (B); ungrafted silica nanoparticles (C); and aligned maghemite nanoparticles (scales are given in $\mathrm{nm}$ ). 